\title{
Role of Factor VII in Correcting Dilutional Coagulopathy and Reducing Re-operations for Bleeding Following Non-traumatic Major Gastrointestinal and Abdominal Surgery
}

\author{
Thomas C. Tsai • James H. Rosing • Jeffrey A. Norton
}

Received: 23 January 2010 /Accepted: 11 May 2010/Published online: 2 June 2010

(C) The Author(s) 2010. This article is published with open access at Springerlink.com

\begin{abstract}
Objective The objective of this study is to evaluate the effectiveness of rfVIIa in reducing blood product requirements and re-operation for postoperative bleeding after major abdominal surgery.

Background Hemorrhage is a significant complication after major gastrointestinal and abdominal surgery. Clinically significant bleeding can lead to shock, transfusion of blood products, and re-operation. Recent reports suggest that activated rfVIIa may be effective in correcting coagulopathy and decreasing the need for re-operation.

Methods This study was a retrospective review over a 4-year period of 17 consecutive bleeding postoperative patients who received rfVIIa to control hemorrhage and avoid re-operation. Outcome measures were blood and clotting factor transfusions, deaths, thromboembolic complications, and number of re-operations for bleeding.

Results Seventeen patients with postoperative hemorrhage following major abdominal gastrointestinal surgery (nine pancreas, four sarcoma, two gastric, one carcinoid, and one fistula) were treated with rfVIIa. In these 17 patients, rfVIIa was administered for 18 episodes of bleeding (dose 2,400-9,600 mcg, 29.8-100.8 mcg $/ \mathrm{kg}$ ). Transfusion requirement of pRBC and FFP were each significantly less than pre-rfVIIa. Out of the 18 episodes, bleeding was controlled in 17 (94\%) without surgery, and only one patient returned to the operating room for hemorrhage. There were no deaths and two thrombotic complications. Coagulopathy was corrected by rfVIIa from 1.37 to $0.96(p<0.0001)$.

Conclusion Use of rfVIIa in resuscitation for hemorrhage after non-traumatic major abdominal and gastrointestinal surgery can correct dilutional coagulopathy, reducing blood product requirements and need for re-operation.
\end{abstract}

Keywords Reoperation · Factor VII · Postoperative complications $\cdot$ General surgery $\cdot$ Coagulopathy

\section{Introduction}

Activated factor VII (rfVIIa) plays a key role in hemostasis by activating the extrinsic pathway of coagulation. Factor VIIa initiates coagulation by interacting with exposed tissue

T. C. Tsai $\cdot$ J. H. Rosing $\cdot$ J. A. Norton

Department of Surgery, Stanford University School of Medicine,

Stanford, CA, USA

J. A. Norton $(\bowtie)$

300 Pasteur Drive H3591,

Stanford, CA 94305-5641, USA

e-mail: janorton@stanford.edu factor, in turn activating factor $\mathrm{X}$ to produce thrombin. ${ }^{1}$ Thrombin then causes a conformational change of circulating platelets, and the activated surface of platelets further activates the coagulation cascade to produce stable fibrin clots. $^{2}$ Injured sub-endothelial cells express tissue factor. Activation of factor VII by exposed tissue factor occurs at sites of local tissue injury and endothelial damage, making factor VII an attractive therapeutic agent for active hemorrhage following surgical injury.

In 1999, the US Food and Drug administration approved the use of recombinant rfVIIa for the treatment of the inherited bleeding disorders hemophilia A and B as well as inhibitors to factor VIII or IX. Because rfVIIa targets areas of active bleeding, it has been used to treat nonhemophiliac cases of coagulopathy or refractory hemorrhage in trauma, neurosurgery, cardiac surgery, and liver transplantation. ${ }^{3,4}$ In randomized, placebo-controlled trials 
among trauma patients, rfVIIa was shown to significantly decrease units of red blood cells (RBC) transfused. ${ }^{5,6}$ Multiple case reports and case series have since suggested the effectiveness of rfVIIa in treating hemorrhage during surgery. ${ }^{7-10}$

Although these studies have shown significant reductions in bleeding, there are no studies clearly evaluating the role of rfVIIa in preventing re-operations for hemorrhage following major abdominal gastrointestinal surgical procedures. Although sepsis and anastamotic leaks are the most common indications for re-operation following gastrointestinal surgery, bleeding accounts for as much as $18.5 \%$ of urgent abdominal re-explorations. ${ }^{11,12}$ Because these reoperations represent additional trauma to the patient and family and may result in increased morbidity and mortality, surgeons are faced with the dilemma of continuing potentially futile resuscitation with blood products or performing a potentially harmful repeat operation. In general, in these situations re-operation has been recommended. However, we hypothesized that rfVIIa would control bleeding by correcting dilutional coagulopathy after surgery without significant complications. To evaluate the role of rfVIIa in preventing re-operations for hemorrhage following major abdominal and gastrointestinal surgery, we performed a retrospective chart review of 17 consecutive patients treated by a single surgeon with rfVIIa to avoid reoperation.

\section{Methods}

This is a retrospective case series from a single surgeon in a large tertiary referral medical center who prospectively planned to first use rfVIIa (rather than return immediately to the operating room) if significant bleeding occurred in the postoperative setting following major gastrointestinal and abdominal surgery. Postoperative patients were identified by surgical residents who notified the attending surgeon that there was significant bleeding and may need to return to the operating room (OR). Instead, each patient received rfVIIa for attempted control of hemorrhage without surgery. Inclusion criteria included patients with bleeding less than 7 days from date of operation, 24-h blood loss greater than 4 units packed red blood cells (pRBC), and clinical evidence of hemorrhagic shock (systolic blood pressure $<100 \mathrm{~mm} \mathrm{Hg}$, pulse $>100$, and urine output $<400 \mathrm{cc} / 24 \mathrm{~h}$ ). There was no threshold for Jackson-Pratt drain output, but increasing output and frank blood instead of serosanguinous output were considered by surgical residents as evidence of postoperative hemorrhage. Exclusion criteria included postoperative blood loss occurring greater than 7 days from date of surgery and patients receiving prophylactic or intraoperative rfVIIa. Patient records from November 2004-November 2008 were reviewed, and 17 patients who met these criteria were identified.

The primary outcome was whether a patient needed surgical re-exploration for hemorrhage within 3 days following administration of rfVIIa. Secondary outcomes included units of packed red blood cells, fresh frozen plasma, cryoprecipitate, and platelets administered. Blood components were calculated as total units in the 24-h period prior to and following rfVIIa administration. Postoperative blood loss was calculated by subtracting immediate postoperative hematocrit by immediate pre-rfVIIa hematocrit. This should be considered as an approximation of actual postoperative blood loss as peri-operative fluid shifts, IV fluid administration, and variability in timing of lab samples preclude definitive characterization of perioperative blood loss based on this calculation. Additionally, mortality and thromboembolic complications following administration of rfVIIa were assessed. Diagnostic criteria for thromboembolic complications included diagnosis of a lower extremity deep venous thrombosis by duplex ultrasound, hemorrhagic or embolic stroke on head CT, acute myocardial infarction by EKG and serial troponin measurement, and limb or mesenteric ischemia. Given the complexity of operations contributing to the sample, we included portal vein or SMV thrombosis diagnosed by CT as thromboembolic complications.

$P$ values reported for transfusion requirements, postoperative blood loss, and coagulopathy were calculated using Wilcoxon rank sum tests. All statistical analyses were performed using SAS 9.2 (SAS Institute, Cary, NC, USA).

This study did not require IRB review and approval because data were extracted from the medical record in a de-identified blinded fashion.

\section{Results}

Seventeen patients with postoperative hemorrhage following major abdominal surgery were treated with activated recombinant factor VII. Out of these 17 patients, nine cases were due to pancreatic tumors requiring a Whipple procedure or a distal pancreatectomy, four cases were due to sarcomas requiring resection of the mass and involved gastrointestinal structures, two cases were due to gastric cancer requiring gastrectomy and Roux-en-Y gastrojejunostomy, one case was due to goblet cell carcinoid requiring a right hemicolectomy, and one case was due to chylous ascites requiring laparotomy and ligation of the cisterna chyli (Table 1). Average case length was $252 \mathrm{~min}$. Average estimated blood loss was $1.5 \mathrm{~L}$, requiring $5.1 \pm$ $0.8 \mathrm{~L}$ crystalloid,. $8 \pm 0.2 \mathrm{~L}$ colloid, $3.5 \pm 1.6$ units pRBC, and 1.6 \pm 1.0 units fresh frozen plasma (FFP; Table 2). The 
Table 1 Clinical Characteristics

\begin{tabular}{|c|c|c|c|c|c|c|}
\hline Age & Weight & Diagnosis & Primary procedure & $\begin{array}{l}\text { Thromboembolic } \\
\text { complications }\end{array}$ & Mortality & $\begin{array}{l}\text { Re-operation } \\
\text { for bleeding }\end{array}$ \\
\hline 32.00 & 66.6 & $\begin{array}{l}\text { Left flank Ewing's } \\
\text { sarcoma }\end{array}$ & $\begin{array}{l}\text { Resection of mass +hernia repair } \\
\text { with mesh and latissimus, } \\
\text { gluteal, and paraspinous muscle } \\
\text { flaps }\end{array}$ & No & No & Yes \\
\hline 74.00 & 94 & IPMT & Whipple & No & No & No \\
\hline 52.00 & 52.3 & $\begin{array}{l}\text { Pancreatic cyst, } \\
\text { pancreatitis }\end{array}$ & $\begin{array}{l}\text { Pancreatic neck dissection } \\
\text { with Roux-en-Y } \\
\text { pancreaticojejunostomy }\end{array}$ & No & No & No \\
\hline 56.00 & 92.7 & $\begin{array}{l}\text { Adenocarcinoma of the } \\
\text { duodenum, sigmoid } \\
\text { colon }\end{array}$ & $\begin{array}{l}\text { Pancreaticoduodenectomy, } \\
\text { sigmoid resection with rectal } \\
\text { anastamosis. SMV injured } \\
\text { intraop and reconstructed. }\end{array}$ & Yes & No & No \\
\hline 69.00 & 69.1 & $\begin{array}{l}\text { Cystic mucinous } \\
\text { tumor }\end{array}$ & $\begin{array}{l}\text { Distal pancreatectomy, } \\
\text { splenectomy }\end{array}$ & No & No & No \\
\hline 53.00 & 78.2 & $\begin{array}{l}\text { Intra-abdominal } \\
\text { liposarcoma }\end{array}$ & Resection of mass & No & No & No \\
\hline 47.00 & 50 & $\begin{array}{l}\text { Left buttock neurofibroma } \\
\text { with AV malformation } \\
\text { and hematoma }\end{array}$ & Resection of mass & No & No & No \\
\hline 49.00 & 72.7 & Chylous ascites & $\begin{array}{l}\text { Laparotomy, ligation of } \\
\text { cisterna chyli }\end{array}$ & No & No & No \\
\hline 67.00 & 79.5 & $\begin{array}{l}\text { Serous cystadenoma } \\
\text { of pancreas }\end{array}$ & $\begin{array}{l}\text { Distal pancreatectomy and } \\
\text { splenectomy }\end{array}$ & No & No & No \\
\hline 72.00 & 40.9 & $\begin{array}{l}\text { Gastric outlet obstruction } \\
\text { with afferent loop syndrome }\end{array}$ & $\begin{array}{l}\text { Resection of Bilroth II } \\
\text { gastrojejunostomy with } \\
\text { new Roux-en-Y gastro } \\
\text { jejunostomy and jejunal } \\
\text { tube }\end{array}$ & No & No & No \\
\hline 85.00 & 76.4 & Retroperitoneal liposarcoma & $\begin{array}{l}\text { Resection of mass, Left } \\
\text { hemicolectomy }\end{array}$ & No & No & No \\
\hline 76.00 & 49.9 & $\begin{array}{l}\text { Goblet cell carcinoid tumor } \\
\text { appendix, intraabdominal } \\
\text { abscess }\end{array}$ & Right hemicolectomy & No & No & No \\
\hline 63.00 & 74 & Pancreatic NET & $\begin{array}{l}\text { Pancreaticoduodenectomy } \\
\text { with SMA and SMV } \\
\text { reconstruction }\end{array}$ & Yes & No & No \\
\hline 71.00 & 47.6 & $\begin{array}{l}\text { Mucinous cystic neoplasm } \\
\text { pancreas }\end{array}$ & Pancreaticoduodenectomy & No & No & No \\
\hline 76.00 & 56 & IPMT & $\begin{array}{l}\text { Laparotomy, subtotal } \\
\text { pancreatectomy, } \\
\text { splenectomy, partial } \\
\text { gastrectomy, transverse } \\
\text { colectomy }\end{array}$ & No & No & No \\
\hline 78.00 & 57.2 & Gastric cancer & $\begin{array}{l}\text { Total gastrectomy, } \\
\text { roux-en-y }\end{array}$ & No & No & No \\
\hline 88.00 & 50.8 & Pancreatic cancer & $\begin{array}{l}\text { Distal pancreatectomy, } \\
\text { splenectomy, L nephrectomy }\end{array}$ & No & No & No \\
\hline
\end{tabular}

high volume of intraoperative crystalloid and $\mathrm{pRBC} / \mathrm{FFP}$ may have contributed to postoperative dilutional coagulopathy as immediate postoperative hematocrit was $30.4 \pm 1.5$.

In $16 / 17$ patients, there was a single episode of bleeding occurring within 7 days of the operation. One patient had two episodes of bleeding in the defined post-operative period; therefore, rfVIIa was administered for 18 episodes of bleeding (doses 2,400-9,600 mcg, 29.8-100.8 mcg/ $\mathrm{kg}$ ). The patient who had two episodes of bleeding was a 74- year-old female undergoing Whipple pancreaticoduodenectomy for an intraductal papillary mucinous tumor who had bleeding on post-operative days 0 and 2 . In all cases, there was clinical evidence of hemorrhage necessitating resuscitation with blood component products and the possibility of return to the OR. Postoperatively, average blood loss resulted in a reduction of the hematocrit from a 30.4 postoperatively to 22.6 when the decision for rfVIIa was made (Table 3). Post-rfVIIa hematocrit of $32.1 \quad(p<$ 
Table 2 Operative Characteristics

\begin{tabular}{|c|c|c|c|c|c|c|c|c|}
\hline Patient & $\begin{array}{l}\text { Duration of } \\
\text { case (min) }\end{array}$ & $\begin{array}{l}\text { Intraoperative } \\
\text { EBL }\end{array}$ & $\begin{array}{l}\text { Intraoperative } \\
\text { crystalloid }\end{array}$ & $\begin{array}{l}\text { Intraoperative } \\
\text { colloid }\end{array}$ & $\begin{array}{l}\text { Intraoperative } \\
\text { pRBC }\end{array}$ & $\begin{array}{l}\text { Intraoperative } \\
\text { FFP }\end{array}$ & $\begin{array}{l}\text { ASA } \\
\text { class }\end{array}$ & $\begin{array}{l}\text { Immediate } \\
\text { postoperative Hct }\end{array}$ \\
\hline 1 & 304 & 4,500 & 7,000 & 250 & 8 & 2 & 3 & 31.0 \\
\hline 2 & 396 & 1,000 & 4,200 & 1,000 & 0 & 0 & 2 & 35.2 \\
\hline 3 & 315 & 500 & 4,200 & 0 & 0 & 0 & 2 & 29.0 \\
\hline 4 & 237 & 1,200 & 11,000 & 1,250 & 4 & 0 & 4 & 35.2 \\
\hline 5 & 150 & 250 & 4,000 & 0 & 0 & 0 & 3 & 32.7 \\
\hline 6 & 288 & 700 & 8,000 & 2,000 & 2 & 0 & 3 & 28.8 \\
\hline 7 & 108 & 250 & 1,200 & 500 & 0 & 0 & 2 & 14.6 .0 \\
\hline 8 & 180 & 500 & 5,700 & 1,000 & 2 & 2 & 3 & 32.2 \\
\hline 9 & 245 & 1,000 & 8,000 & 1,500 & 2 & 0 & 2 & 31.2 \\
\hline 10 & 203 & 200 & 3,000 & 500 & 0 & 0 & 3 & 36.4 \\
\hline 11 & 270 & 3,000 & 6,000 & 3,500 & 10 & 2 & 4 & 41.0 \\
\hline 12 & 140 & 250 & 800 & 0 & 2 & 0 & 3 & 33.5 \\
\hline 13 & 535 & 8,000 & 13,000 & 1,000 & 26 & 17 & 2 & 28.7 \\
\hline 14 & 324 & 200 & 2,500 & 1,000 & 0 & 0 & 3 & 24.6 \\
\hline 15 & 269 & 1,900 & 4,400 & 1,000 & 4 & 4 & 2 & 21.2 \\
\hline 16 & 162 & 2,000 & 0 & 0 & 0 & 0 & 2 & 28.5 \\
\hline 17 & 158 & 250 & 4,000 & 0 & 0 & 0 & 3 & 33.1 \\
\hline Median & 245 & 700 & 4,200 & 1,000 & 2 & 0 & 3 & 31.2 \\
\hline Mean & 252 & 1,512 & 5,118 & 853 & 3.5 & 1.6 & 3 & 30.4 \\
\hline $\begin{array}{l}\text { Standard } \\
\text { error }\end{array}$ & 25.9 & 495.4 & 845.0 & 220.8 & 1.6 & 1.0 & 0.2 & 1.5 \\
\hline
\end{tabular}

Fluids are in milliliter. Blood components are in units transfused

ASA American Society of Anesthesiologist

$0.00001)$ was significantly higher than pre-rfVIIa. There was also a significant reduction in the INR $(p<0.01)$.

In the $24 \mathrm{~h}$ pre-rfVIIa administration, a mean of 5.2 units pRBCs, 2.0 units FFP, 33 units cryoprecipitate, and 0.39 units platelets were administered. Median values for pRBC, FFP, cryoprecipitate, and platelets were 4, 1.5, 0, and 0 , respectively. In the $24 \mathrm{~h}$ post-rfVIIa administration, a mean of 0.83 units pRBC, 0.38 units FFP, 0 units Cryo, and 0.06 units platelets were given. Median values for all blood products post-rfVIIa were 0 (Table 4). Transfusion of pRBC and FFP was significantly less than pre-rfVIIa amount by Wilcoxon rank sum test $(p<0.00001$ and $p<0.01)$, and transfusion of cryoprecipitate and platelets were not significantly different ( $p=0.06$ and $p=0.09$ ).

Out of the 18 episodes, bleeding was immediately controlled in 17 (94\%) without surgery. One patient returned to the OR for surgical control of bleeding (retroperitoneal hemorrhage following flank sarcoma resection and hernia repair). There were no deaths. There were two thrombotic complications involving major veins, one with thrombosis of the superior mesenteric vein and another with thrombosis of the portal vein. Both of these veins had been partially reconstructed during the surgical procedure. Neither patient developed long-term complications from the

Table 3 Postoperative Blood Loss and Coagulopathy Corrected by rfVIIa

\begin{tabular}{lcccc}
\hline & Postop Blood Loss & Pre-FVII & Post-FVII & $p$ \\
\hline Hematocrit & $-7.8(-7)$ & $22.6(22.7)$ & $32.1(32.6)$ & $<2(142)$ \\
Platelets & - & $212(188)$ & $172\left(0.00001^{\mathrm{a}}\right.$ \\
INR & - & $1.37(1.4)$ & $0.96(0.9)$ & 0.40 \\
\hline
\end{tabular}

Units reported are mean and (median)

Unit for hematocrit is $\%$ of blood volume occupied by red blood cells. Unit for platelets is $10^{3} / \mathrm{uL}$

${ }^{\text {a }}$ Statistically significant 
Table 4 Transfusion Requirements pre- and post-rfVIIa Administration

$p R B C$ packed red blood cell, $\overline{F F P}$ fresh frozen plasma, Cryo cryoprecipitate

${ }^{\text {a }}$ Statistically significant

\begin{tabular}{lccc}
\hline & 24h pre-factor VIIa, mean (median) units & 24h post-factor VII (median units) & $P$ value \\
\hline pRBC & $5.2(4)$ & $0.83(0)$ & $<0.00001^{\mathrm{a}}$ \\
FFP & $2(1.5)$ & $0.38(0)$ & $<0.01^{\mathrm{a}}$ \\
Cryo & $0.33(0)$ & $0(0)$ & $=0.06$ \\
Platelet & $0.39(0)$ & $0.06(0)$ & $=0.09$ \\
\hline
\end{tabular}

thrombosis. There were otherwise no cases of stroke, myocardial infarction, or pulmonary embolism. The dose of rfVIIa given ranged from 29.8 to $100.8 \mathrm{mcg} / \mathrm{kg}$, with an average of $78.0 \mathrm{mcg} / \mathrm{kg}$. Bleeding was controlled in $8 / 18$ episodes $(44.4 \%)$ with the initial dose of rfVIIa, while $10 /$ $18(55.6 \%)$ bleeding episodes required two or more doses. We subsequently routinely planned to give two doses separated by $2 \mathrm{~h}$, and in the latter half of the study period (2006-2008), 7/9 (77.8\%) bleeding episodes were treated with two doses.

\section{Discussion}

Current dogma in surgery differentiates between surgical and non-surgical causes of bleeding. As a cause of surgical bleeding, postoperative hemorrhage has been traditionally managed by re-operation. However, reoperation costs a significant amount in money, time, energy, and potential patient and family suffering. This study demonstrates the ability of rfVIIa in conjunction with component resuscitation to control hemorrhage and avoid reoperation for bleeding following complex major abdominal and gastrointestinal surgery.

Postoperative hemorrhage following major abdominal surgical procedures such as pancreatectomy and pancreatoduodenectomy, the most common operations in our case series, represent a challenging complication, with a mortality rate as high as $43 \%{ }^{13}$ Likewise, for gastrointestinal procedures such as gastrectomies, reoperation for postoperative bleeding has also been shown to be associated with increased hospital length of stay as well as morbidity. ${ }^{14}$ The primary intervention for control of post-operative hemorrhage is ether transfusion of blood products or re-operation. From a review of the literature, in $47.2-69 \%$ of cases blood product transfusion alone was unable to cease hemorrhage, necessitating urgent surgical re-exploration for hemostatic control. $^{13,15-17}$ However, both surgical re-exploration and excessive blood product replacement have been associated with increased infection risk, multiorgan dysfunction, increased hospital length of stay, and mortality. ${ }^{11-13,18-20}$ More recently, interventional radiology has been used for treatment of postoperative hemorrhage, but in terms of hemostasis, morbidity, and mortality, it is comparable to surgical re-exploration. ${ }^{13}$ Therefore, reducing the amount of blood products transfused and avoiding a re-operation presents a real benefit to patents.

The administration of rfVIIa was effective in arresting hemorrhage and preventing re-operation in $17 / 18$ episodes $(94.4 \%)$. Further, it resulted in a reduction of blood products transfused for all patients. Additionally, our results show that rfVIIa administration was associated minimal morbidity. Given that the average patient in our series had a class 3 physical status by the American Society of Anesthesiologist, reoperation would present increased risks.

Although there are many studies evaluating the use of rfVIIa in the perioperative setting, ${ }^{21,22}$ there are none that evaluate the use of rfVIIa specifically in the postoperative setting in major gastrointestinal and abdominal cases. ${ }^{8,10}$ Most previous reports have focused on vascular and cardiac surgery. $^{23-25}$ Because the current study excluded any patient who received prophylactic and intra-operative rfVIIa, we were able to assess the role of rfVIIa to prevent re-operation in the postoperative setting for postoperative hemorrhage, the primary outcome measurement of this study. Administration of rfVIIa appeared to be an effective therapy as it prevented re-operation in $94.4 \%$ of cases, normalized coagulopathy, and raised the hematocrit. The single case that returned to the OR for hemostatic control involved a patient undergoing a complicated operation involving resection of a large left flank Ewing's sarcoma; hernia repair with mesh; and latissimus, gluteal, and paraspinous muscle flaps. In this patient, we wanted to avoid a hematoma that would have compromised the integrity of the hernia repair with mesh and flaps.

Administration of rfVIIa also significantly reduced blood transfusion requirement for red blood cells, fresh frozen plasma, cryoprecipitate, and platelets. This is a similar finding to other studies that have evaluated the efficacy of rfVIIa in controlling hemorrhage among trauma, cardiac, vascular, and liver cases. ${ }^{3,5,6,26,27}$ The average dose of rfVIIa was $78.0 \mathrm{mcg} / \mathrm{kg}$, and this is consistent with recent studies showing efficacy of doses of $\mathrm{rfVIIa}>50 \mathrm{mcg} / \mathrm{kg}^{28}$ However, the wide range of dosing among various studies occurs because of limited initial experience with the drug. Eventually, the dose for rfVIIa was standardized to $>50 \mathrm{mcg} / \mathrm{kg}$ for two doses, and this was done in the last ten patients.

Thrombosis is a potential complication of this drug. Thrombosis of two major veins that had been reconstructed 
was observed. It is unclear if the thrombosis was a complication of the procedure itself or to rfVIIa. However, both patients stopped bleeding without reoperation and neither patient had any long-term sequellae from the thrombosis. There is a lack of consensus in the literature regarding the association of rfVIIa with thromboembolic complications with some studies concluding an increased association $^{3}$ while others conclude no association. ${ }^{7,28}$ In a meta-analysis of rfVIIa used peri-operatively among abdominal surgery patients, rfVIIa was not observed to increase thromboembolic risk. Nevertheless, thromboembolic complications should be considered, and the surgeon must weigh the benefits of avoiding re-operation to the potential risk of thromboembolism. Based on its efficacy and the limited morbidity, we have continued to choose rfVIIa to control postoperative bleeding and avoid re-operation.

Given the high cost of rfVIIa, other staff surgeons and pharmacists have been cautious about its use. Using the standard pharmacy Red Book reference and pharmacy department at our institution, the price of rfVIIa is $\$ 1.08$ per mcg, supplied in 1,$200 ; 2,400$; and 4,800 mcg vials. For a $4,800-\mathrm{mcg}$ dose $(68.6 \mathrm{mcg} / \mathrm{kg}$ for a $70-\mathrm{kg}$ patient), the purchasing cost is $\$ 5,184$. Patient charges at our institution for $2 \mathrm{~h}$ of OR time is $\$ 17,640$, aggregating anesthesia, surgery, and OR time charges. Although a full costeffectiveness analysis was not undertaken as part of this retrospective study, a basic cost analysis would suggest that rfVIIa should be considered when surgical re-exploration is the other alternative given the high cost of re-operation. Further, we did not include the cost of continued transfusion of blood and clotting factors that the use of rfVIIa effectively reduced. Although this was not included in our analysis, transfusion of blood products carry significant risk for infections and increased length of stay, also arguing for the use of rfVIIa to avoid reoperation. One cost-benefit analysis has suggested that rfVIIa is only favorable if the patient is expected to receive 40 units of $\mathrm{RBC}$ or one whole blood transfusion (RBC, clotting factors, and platelets). ${ }^{28}$ Loudon and Smith have proposed that rfVIIa is costeffective after transfusion of 14 units of RBC. ${ }^{29}$ Including the averted cost of re-operation, the cost of additional blood products plus the cost of other related complications would elucidate whether rfVIIa is potentially cost saving in the setting of postoperative hemorrhage. Our results suggest that a full cost-effectiveness analysis using decision analytic models would provide important information for surgeons and pharmacists when deciding to use rfVIIa.

Algorithms for the use of rfVIIa for postoperative hemorrhage following non-traumatic major abdominal and gastrointestinal surgery need to be developed. Clark and colleagues defined three criteria for the administration of rfVIIa: (1) transfusions of RBC to at least 1.5 -fold the blood volume ( $>15$ units), (2) persistence of bleeding despite conventional therapy, and (3) no foreseeable immediate surgical bleeding control. ${ }^{30}$ However, this algorithm ignores the benefit posed by preventing the high morbidities associated with surgical re-operation, which is consistently recommended in the surgical literature. Instead, Von Heymann and colleagues suggest that in situations of persistent massive postoperative bleeding where the remaining options are only rfVIIa or surgical re-exploration, a $90 \mathrm{mcg} / \mathrm{kg}$ of rfVIIa should be given, repeated by another dose one hour later if bleeding persists. ${ }^{7}$ This algorithm implies that successful treatment with rfVIIa must be accompanied by correction of acidosis $(\mathrm{pH}>7.2)$, fibrinogen $(>100 \mathrm{mg} / \mathrm{dL})$, platelets $(>50,000 / \mathrm{uL})$, and hematocrit $(>24 \%)$, each of which may impact the efficacy of rfVIIa. ${ }^{31}$ We also have tried to correct these factors before administering rfVIIa perhaps contributing to the effectiveness of rfVIIa for preventing re-operation in our experience.

Factor VII has been extensively studied among the trauma surgery literature. ${ }^{5,6,32}$ Additionally, the role of increased FFP to PRBC ratio has also been examined in massive transfusion protocols for civilian trauma. ${ }^{33,34} \mathrm{~A}$ massive transfusion protocol was implemented at our institution in July 2005 supporting a 1:1.5 FFP/PRBC ratio, improved communications, and enhanced systems flow to optimize rapid blood product availability. Activation is recommended for greater than 4 units PRBCs transfused in the first hour or expected transfusion requirements in excess of 10 units in a 12-h period. In our clinical algorithm, factor VII is recommended for consideration if there is persistent coagulopathy or hemorrhage after administration of two massive transfusion packs or 12 units pRBCS and 8 units of FFP.

Studies have shown that civilian trauma patients undergoing massive resuscitation often arrive with significant dilutional coagulopathy from excessive crystalloid administration. ${ }^{35}$ Likewise, the patients in our sample may have also had significant dilutional coagulopathy as they received a large amount of intraoperative crystalloid, with a mean of $5.1 \mathrm{~L}$ resulting in mean immediate postoperative hematocrit of 30.4. They received relatively few intraoperative pRBC ( $3.5 \pm 1.6$ units) and FFP ( $1.6 \pm 1.0$ units) given the large estimated blood loss $(1,512 \pm 495.4 \mathrm{cc})$ for a $\mathrm{pRBC} / \mathrm{FFP}$ ratio of 2:2. Although the interpretation of these data is challenged by the small sample size and large standard error, these findings nevertheless highlight that the patients in this sample could have had significant dilution of clotting factors secondary to large intraoperative crystalloid and high pRBC relative to FFP transfusion. This may be a common problem among patients undergoing complex and long gastrointestinal and tumor resection surgical procedures. This dilutional coagulopathy may explain the success of rfVIIa in stopping bleeding in our patients. 
Implementation of our massive transfusion protocol (MTP) among trauma patients at our institution has been shown to improve mortality. ${ }^{36}$ Given that the trauma literature suggests improved outcomes with a 1:1 ratio of FFP to pRBC, perhaps the need for rfVIIa could have been averted had a 1:1 pRBC/FFP ratio been administered instead of the ratio of 2:2 intra-operatively and 2:6 postoperatively in this series. However, this is complicated by data from our institution showing increased survival due to decreased time to transfusion, as component ratios did not change in the periods prior to and post-implementation of the MTP. ${ }^{36}$ Because our review extended to patients in 2004, five patients were resuscitated prior to the advent of our institution's massive transfusion protocol. Additionally, the patients in this study did not meet our criteria for MTP, since average blood loss in the 24-h period prior to rfVIIa administration was 5.2 units pRBC. Therefore, for the patients in our series, the amount and endpoints of component resuscitation and rfVIIa occurred as a result of direct decision making of the surgeon and not as a consequence of the institutional MTP. This study suggests the inadequacy of applying trauma resuscitation protocols to patients undergoing major abdominal tumor resections as patient characteristics such as age, type of operation, and comorbidities clearly differ. Clinical decision-making algorithms should be derived specifically for the setting of postoperative bleeding and coagulopathy following nontraumatic abdominal and gastrointestinal procedures.

Deficiencies of this study include small sample size $(n=17)$ and study design (retrospective case series). Similar to previous studies of the use of rfVIIa among surgical patients, a more robust case-control analysis was not possible because of the heterogeneity of diagnoses and operations. ${ }^{8}$ An advantage of this study was that there was no operator variability in surgical technique or clinical decision making, since all patients were under the care of one surgeon. In each instance if rfVIIa had not been used, the attending surgeon deemed that the patient would have needed to undergo surgical re-exploration.

Currently, there are no indications for the administration rfVIIa for hemorrhage following major abdominal and gastrointestinal surgery in the absence of inherited coagulation disorders. However, this and other studies have suggested that rfVIIa reduces blood transfusion requirements, posing both clinical and pharmaco-economic benefit. Additionally, rfVIIa should be considered as an alternative to re-operation for surgical control of bleeding in cases of postoperative hemorrhage refractory to blood products. However, thromboembolic complications may be a risk of this approach.

Randomized controlled studies and evaluations of clinical algorithms are needed to provide a prospective assessment of the role of rfVIIa in the common surgical challenge of postoperative bleeding. This study highlights that for patients undergoing long and complex gastrointestinal surgical procedures, dilutional coagulopathy from intraoperative resuscitation may be an under-recognized condition that exacerbates postoperative bleeding. For this subset of patients, rfVIIa in conjunction with traditional blood component resuscitation can control bleeding and decrease the probability of return to the OR in patients for whom re-operation would pose significant risk of complications. However, given the small sample size and limitations of this study, further research is warranted to describe the subset of patients who would benefit most and the cost-effectiveness of this approach.

Open Access This article is distributed under the terms of the Creative Commons Attribution Noncommercial License which permits any noncommercial use, distribution, and reproduction in any medium, provided the original author(s) and source are credited.

\section{References}

1. Mannucci PM, Levi M. Prevention and treatment of major blood loss. N Engl J Med 2007;356(22):2301-2311.

2. Hoffman M, Monroe DM, 3rd, Roberts HR. Activated factor VII activates factors IX and X on the surface of activated platelets: thoughts on the mechanism of action of high dose-activated factor VII. Blood Coagul Fibrinolysis 1998;9(Suppl 1):S61-S65.

3. Stanworth SJ, Birchall J, Doree CJ, Hyde C. Recombinant factor VIIa for the prevention and treatment of bleeding in patients without haemophilia. Cochrane Database Syst Rev. 2007(2): CD005011.

4. Levi M, Peters M, Buller HR. Efficacy and safety of recombinant factor VIIa for treatment of severe bleeding: a systematic review. Crit Care Med 2005;33(4):883-890.

5. Boffard KD, Riou B, Warren B, et al. Recombinant factor VIIa as adjunctive therapy for bleeding control in severely injured trauma patients: two parallel randomized, placebo-controlled, doubleblind clinical trials. J Trauma 2005;59(1):8-15. discussion 15-18.

6. Rizoli SB, Boffard KD, Riou B, et al. Recombinant activated factor VII as an adjunctive therapy for bleeding control in severe trauma patients with coagulopathy: subgroup analysis from two randomized trials. Crit Care 2006;10(6):R178.

7. von Heymann C, Jonas S, Spies C, et al. Recombinant activated factor VIIa for the treatment of bleeding in major abdominal surgery including vascular and urological surgery: a review and meta-analysis of published data. Crit Care 2008;12 (1):R14.

8. Benharash P, Bongard F, Putnam B. Use of recombinant factor VIIa for adjunctive hemorrhage control in trauma and surgical patients. Am Surg 2005;71(9):776-780.

9. Wordliczek J, Serednicki W, Grabowska I, Szczepanik AM, Nowak W, Popiela T. A 43-year-old man was admitted to the hospital with the diagnosis of acute pancreatitis of 4 days' duration. Pancreas 2003;26(1):101-102.

10. Vlachos K, Archontovasilis F, Papadima A, et al. Successful use of recombinant activated factor VII for postoperative associated haemorrhage: a case report. Cases J 2008;1(1):361.

11. Harbrecht PJ, Garrison RN, Fry DE. Early urgent relaparotomy. Arch Surg 1984;119(4):369-374. 
12. Unalp HR, Kamer E, Kar H, Bal A, Peskersoy M, Ali Onal M. Urgent abdominal re-explorations. World J Emerg Surg 2006;1:10

13. Limongelli P, Khorsandi SE, Pai M, et al. Management of delayed postoperative hemorrhage after pancreaticoduodenectomy: a metaanalysis. Arch Surg 2008;143(10):1001-1007. Discussion 1007

14. Sah BK, Chen MM, Yan M, Zhu ZG (2010) Reoperation for early postoperative complications after gastric cancer surgery in a Chinese hospital. World J Gastroenterol 16(1):98-103.

15. Wente MN, Shrikhande SV, Kleeff J, et al. Management of early hemorrhage from pancreatic anastomoses after pancreaticoduodenectomy. Dig Surg 2006;23(4):203-208.

16. Yekebas EF, Wolfram L, Cataldegirmen G, et al. Postpancreatectomy hemorrhage: diagnosis and treatment: an analysis in 1669 consecutive pancreatic resections. Ann Surg 2007;246(2):269280.

17. Rumstadt B, Schwab M, Korth P, Samman M, Trede M. Hemorrhage after pancreatoduodenectomy. Ann Surg 1998;227 (2):236-241.

18. Moore FA, Moore EE, Sauaia A. Blood transfusion. An independent risk factor for postinjury multiple organ failure. Arch Surg 1997;132(6):620-624. Discussion 624-625

19. Taylor RW, Manganaro L, O'Brien J, Trottier SJ, Parkar N, Veremakis C. Impact of allogenic packed red blood cell transfusion on nosocomial infection rates in the critically ill patient. Crit Care Med 2002;30(10):2249-2254.

20. Blajchman MA, Vamvakas EC. The continuing risk of transfusion-transmitted infections. N Engl J Med 2006;355 (13):1303-1305.

21. Friederich PW, Geerdink MG, Spataro M, et al. The effect of the administration of recombinant activated factor VII (NovoSeven) on perioperative blood loss in patients undergoing transabdominal retropubic prostatectomy: the PROSE study. Blood Coagul Fibrinolysis 2000;11(Suppl 1):S129-S132.

22. Friederich PW, Henny CP, Messelink EJ, et al. Effect of recombinant activated factor VII on perioperative blood loss in patients undergoing retropubic prostatectomy: a double-blind placebo-controlled randomised trial. Lancet 2003;361(9353): 201-205.

23. Zangrillo A, Mizzi A, Biondi-Zoccai G, et al. Recombinant activated factor VII in cardiac surgery: a meta-analysis. J Cardiothorac Vasc Anesth 2009;23(1):34-40.

24. Kylasam S, Mos K, Fijtin S, Webster B, Chard R, Egan J. Recombinat activated factor VII following pediatric cardiac surgery. J Intensive Care Med Feb 22009.
25. Bowman LJ, Uber WE, Stroud MR, et al. Use of recombinant activated factor VII concentrate to control postoperative hemorrhage in complex cardiovascular surgery. Ann Thorac Surg 2008;85(5):1669-1676. discussion 1676-1667.

26. O'Connell NM, Perry DJ, Hodgson AJ, O'Shaughnessy DF, Laffan MA, Smith OP. Recombinant FVIIa in the management of uncontrolled hemorrhage. Transfusion 2003;43(12):1711-1716.

27. Duchesne JC, Mathew KA, Marr AB, Pinsky MR, Barbeau JM, McSwain NE. Current evidence based guidelines for factor VIIa use in trauma: the good, the bad, and the ugly. Am Surg 2008;74 (12):1159-1165.

28. Ranucci M, Isgro G, Soro G, Conti D, De Toffol B. Efficacy and safety of recombinant activated factor vii in major surgical procedures: systematic review and meta-analysis of randomized clinical trials. Arch Surg 2008;143(3):296-304; discussion 304.

29. Loudon B, Smith MP. Recombinant factor VIIa as an adjunctive therapy for patients requiring large volume transfusion: a pharmacoeconomic evaluation. Intern Med J 2005;35(8):463-467.

30. Clark AD, Gordon WC, Walker ID, Tait RC. 'Last-ditch' use of recombinant factor VIIa in patients with massive haemorrhage is ineffective. Vox Sang 2004;86(2):120-124.

31. Vincent JL, Rossaint R, Riou B, Ozier Y, Zideman D, Spahn DR. Recommendations on the use of recombinant activated factor VII as an adjunctive treatment for massive bleeding-a European perspective. Crit Care 2006;10(4):R120

32. Spinella PC, Perkins JG, McLaughlin DF, et al. The effect of recombinant activated factor VII on mortality in combat-related casualties with severe trauma and massive transfusion. J Trauma 2008;64(2):286-293. Discussion 293-284

33. Zink KA, Sambasivan CN, Holcomb JB, Chisholm G, Schreiber MA. A high ratio of plasma and platelets to packed red blood cells in the first 6 hours of massive transfusion improves outcomes in a large multicenter study. Am J Surg 2009;197(5):565-570. Discussion 570

34. Holcomb JB, Wade CE, Michalek JE, et al. Increased plasma and platelet to red blood cell ratios improves outcome in 466 massively transfused civilian trauma patients. Ann Surg 2008;248(3):447-458.

35. Levy JH, Dutton RP, Hemphill JC 3rd et al. Multidisciplinary approach to the challenge of hemostasis. Anesth Analg 110 (2):354-364

36. Riskin DJ, Tsai TC, Riskin L, et al. Massive transfusion protocols: the role of aggressive resuscitation versus product ratio in mortality reduction. J Am Coll Surg 2009;209(2):198-205 